\title{
Aplicação de sistemas baseados em microalgas para controle de odores em estações de tratamento de águas residuais: abordagens, avanços e perspectivas
}

Karem Rodrigues Vieira, Pricila Nass Pinheiro, Tatiele Casagrande do Nascimento

https://doi.org/10.4322/mp.978-65-994457-8-1.c4

\section{Resumo}

A emissão de compostos de odores das estações de tratamento de águas residuais tem atraído muita atenção ao longo dos anos e padrões ambientais rigorosos tem sido amplamente adotado para reduzir as emissões. Métodos de tratamento biológico de odores têm aplicações mais amplas do que as contrapartes físicas e químicas, pois são ecológicos, econômicos e geram poucos resíduos secundários. Os sistemas baseados em microalgas estão entre as abordagens mais promissoras para a prevenção ou remoção final de emissões de odores. Além disso, têm o potencial de tratar simultaneamente odores e águas residuais. No entanto, biotecnologias para este fim envolvendo microalgas ainda precisam ser discutidas sobre as características e mecanismos subjacentes do processo de desodorização. Recentemente, estudos consideráveis foram relatados para elucidar o metabolismo microalgal no controle de odores e tratamento de águas residuais. Assim, neste capítulo tópicos como metabolismo microalgal, biossíntese de compostos orgânico voláteis, características e avanços de sistemas baseados em microalgas para tratamento de odores e aplicação de compostos voláteis de microalgas, são discutidos.

Palavras-chave: água residuária, biorreatores, cianobactéria, compostos orgânicos voláteis, poluição gasosa. 


\section{Introdução}

A indústria alimentícia gera grande quantidade de água residuárias durante os seus processos de produção e consequentemente gera um impacto ambiental significativo devido à descarga do efluente com alta concentração de carga orgânica nos corpos d'água, além disso são responsáveis por incômodos olfativos, gerados por diferentes processos químicos ou biológicos durante o tratamento. Os odores são formados principalmente por compostos orgânicos voláteis (COVs) como aldeídos, cetonas, ácidos orgânicos, além de nitrogenados e sulfurados (SADDOUD \& SAYADI, 2007; LEBRERO et al., 2013; LEWKOWSKA et al., 2016).

Compostos orgânicos voláteis são definidos como qualquer composto orgânico cujo ponto de ebulição está na faixa de $\left(50-260^{\circ} \mathrm{C}\right)$, correspondendo a pressões de vapor de saturação maiores que $102 \mathrm{kPa}$ a $25^{\circ} \mathrm{C}$. Uma variedade de COVs são tóxicos, sendo a maioria proveniente de atividades antropogênicas oriundos de emissões veiculares, indústrias petroquímicas e indústrias de manufaturas (EPA 2008; BERENJIAN, CHAN \& MALMIRI, 2012). A exposição humana a COVs de origem antropogênica pode resultar em um espectro de doenças que variam de leves, como irritação, a efeitos muito graves, incluindo câncer (POVEDA, 2021).

Além disso, COVs podem apresentar odores que vai do agradável ao mais intenso e fétido. O odor é certamente o mais complexo de todos os problemas de poluição do ar. A poluição por odor contribui para a formação fotoquímica de fumaça, assim como emissões secundárias de contaminantes. Portanto, é uma ameaça à qualidade do ar, à saúde e bem-estar humano (CAPELLI et al., 2009; BAJPAI, 2014; LEWKOWSKA et al., 2016).

Dentre os métodos utilizados para a mitigação de odor nas estações de tratamento de água residuária estão os processos de absorção, incineração, oxidação, adsorção e tratamento biológico (DOMENO et al., 2010). Métodos biológicos para a remoção de odores e COVs são tecnologias econômicas quando se trata de baixas concentrações, no entanto, suas desvantagens em relação aos poluentes hidrofóbicos têm sido relatadas, além do crescimento excessivo de biofilme e desempenho instável devido às mudanças da comunidade microbiana (BURGESS, PARSONS, STUETZ, 2001; MUNÕZ et al., 2015; CHENG et al., 2021). 
A biotecnologia de microalgas é uma área emergente da tecnologia industrial, que vem se consolidando em função da sua potencialidade de exploração. As microalgas são consideradas uma fonte potencialmente nova e valiosa de compostos biologicamente ativos (NASCIMENTO et al., 2019; PINHEIRO et al., 2019; JACOB-LOPES et a., 2020; SEVERO et al., 2020). Além disso, o uso de microalgas desempenha um papel vital na conversão de resíduos em uma infinidade de produtos, por exemplo, biocombustíveis, nutracêuticos, polímeros, pigmentos, uma variedade de produtos químicos e COVs. Microalgas também apresentam potencial em transformar gases de efeito estufa industriais, bem como águas residuais em produtos úteis, servindo assim como uma plataforma eficaz de captura e utilização de carbono (WANG et al., 2017; JACOB-LOPES et al., 2020).

Por fim, uma inovação tecnológica atual é o uso de microalgas na mitigação de odores liberados de água residuária (VIEIRA et al., 2019; 2021). Assim, este capítulo aborda os tópicos referentes ao metabolismo microalgal, produção de COVs por microalgas, mecanismos de desodorização de água residuária, além do potencial de aplicação de COVs de microalgas.

\section{Microalgas e seu metabolismo}

Microalgas são um grupo de microrganismos fotossintéticos tipicamente unicelulares, eucarióticos ou procarióticos. Desenvolvem-se principalmente em ambientes aquáticos, solos, rochas, e em ambientes extremos, como geleiras e fossas termais, podendo estar associados simbioticamente a outros organismos auxiliando-os na fixação de nitrogênio (HERRERO, MURO-PASTOR, FLORES, 2001; LOURENÇO, 2006; SANTOS et al., 2017).

A diversidade metabólicas destes microarganismos podem explicar sua capacidade em responder rapidamente a alterações no meio onde vivem (ACHYUTHAN et al., 2017). A diversidade deste grupo de microrganismos é destacada por serem responsáveis pela estruturação da atmosfera terrestre, por sua importância ecológica e econômica, sendo que as algas são as maiores removedoras de carbono da biosfera (MOORE, 2001).

Metabolicamente, as espécies de microalgas têm três vias de fixação de carbono: (i) fotoautotrófica, (ii) heterotrófica ou (iii) mixotrófica (PEREZ-GARCIA, \& BASHAN, 2015; SANTOS et al., 2016a). Destes, a via fotoautotrófica é a 
principal rota energética dos microrganismos relacionados (SUGANYA et al., 2016; SEVERO et al., 2019). Este mecanismo envolve o uso de carbono inorgânico $\left(\mathrm{CO}_{2}\right)$ ou íons bicarbonato $\mathrm{HCO}_{3}$ dissolvido em meio aquoso (de acordo com $\mathrm{pH}$ : $\left.\mathrm{CO}_{2}(\mathrm{pH}<5) ; \mathrm{HCO}_{3}^{-}(7<\mathrm{pH}<9)\right)$ como fonte de carbono na presença de luz, principalmente regulado por carbono do metabolismo fotossintético e mecanismos de concentração (KONG, 2021).

Em geral, o metabolismo fotossintético do carbono microalgal ocorre através do ciclo Calvin-Benson-Bassham. Portanto, as microalgas usam energia luminosa para gerar equivalentes redutores e fixar $\mathrm{CO}_{2}$ em moléculas orgânicas (por meio das reações dependentes e independentes de luz) (CALVIN \& BENSON, 1948; SEVERO et al., 2019; SU, 2021). O ciclo de Calvin é composto por 13 etapas catalisadas por cerca de 11 enzimas diferentes e subdivididas em 3 reações: (i) carboxilação, (ii) redução e (iii) regeneração (NOREÑA-CARO \& BENTON, 2018; SEVERO et al., 2020).

Como alternativa à condição de baixa concentração de $\mathrm{CO}_{2}$ viabilizando a fotossíntese, a maioria das microalgas tem diferentes mecanismos de concentração de $\mathrm{CO}_{2}$ : como assimilação de íons $\mathrm{HCO}_{3}{ }^{-}$por meio de transportadores ativos na membrana plasmática; e usando a enzima anidrase carbônica extracelular para conversão aumentada de $\mathrm{HCO}_{3}-$ em $\mathrm{CO}_{2}$ intracelular (NOREÑA-CARO \& BENTON, 2018).

Por outro lado, algumas espécies de microalgas também podem crescer heterotroficamente na ausência de luz, suportadas por uma fonte de carbono exógena. No metabolismo heterotrófico, o substrato é convertido em glicose 6 fosfato para que possa iniciar a via oxidativa da pentose fosfato. Durante 0 metabolismo, ocorre a formação de duas moléculas de ATP (trifosfato de adenosina). O produto final, assim como no cultivo fotossintético, também é o piruvato (SANTOS et al., 2016b; PINHEIRO et al., 2019).

Além disso, algumas espécies de microalgas são mixotróficas e podem causar fototrofia e heterotrofia simultaneamente. Isso porque, o $\mathrm{CO}_{2}$ é fixado pela fotossíntese, enquanto os substratos orgânicos são assimilados pela respiração aeróbia (PEREZ-GARCIA \& BASHAN, 2015; PINHEIRO et al., 2019). 


\section{Mecanismos de formação de compostos orgânicos voláteis em microalgas}

Carbono exógenos são metabolizados a piruvato ou acetil-CoA. Além disso, as vias biossintéticas e metabólicas das microalgas podem converter esses substratos em COVs como terpenos, álcoois, cetonas, aldeídos, ésteres, hidrocarbonetos, ácidos carboxílicos e compostos sulfurados. Em geral, a produção desses compostos é alcançada por meio das vias de 2-cetoácidos, isoprenóides e derivados de ácidos graxos (ZARGAR et al., 2017; JACOBLOPES et al., 2020; SEVERO et al., 2020; VIEIRA, PINHEIRO \& ZEPKA, 2020) como apresentada na Figura 1.

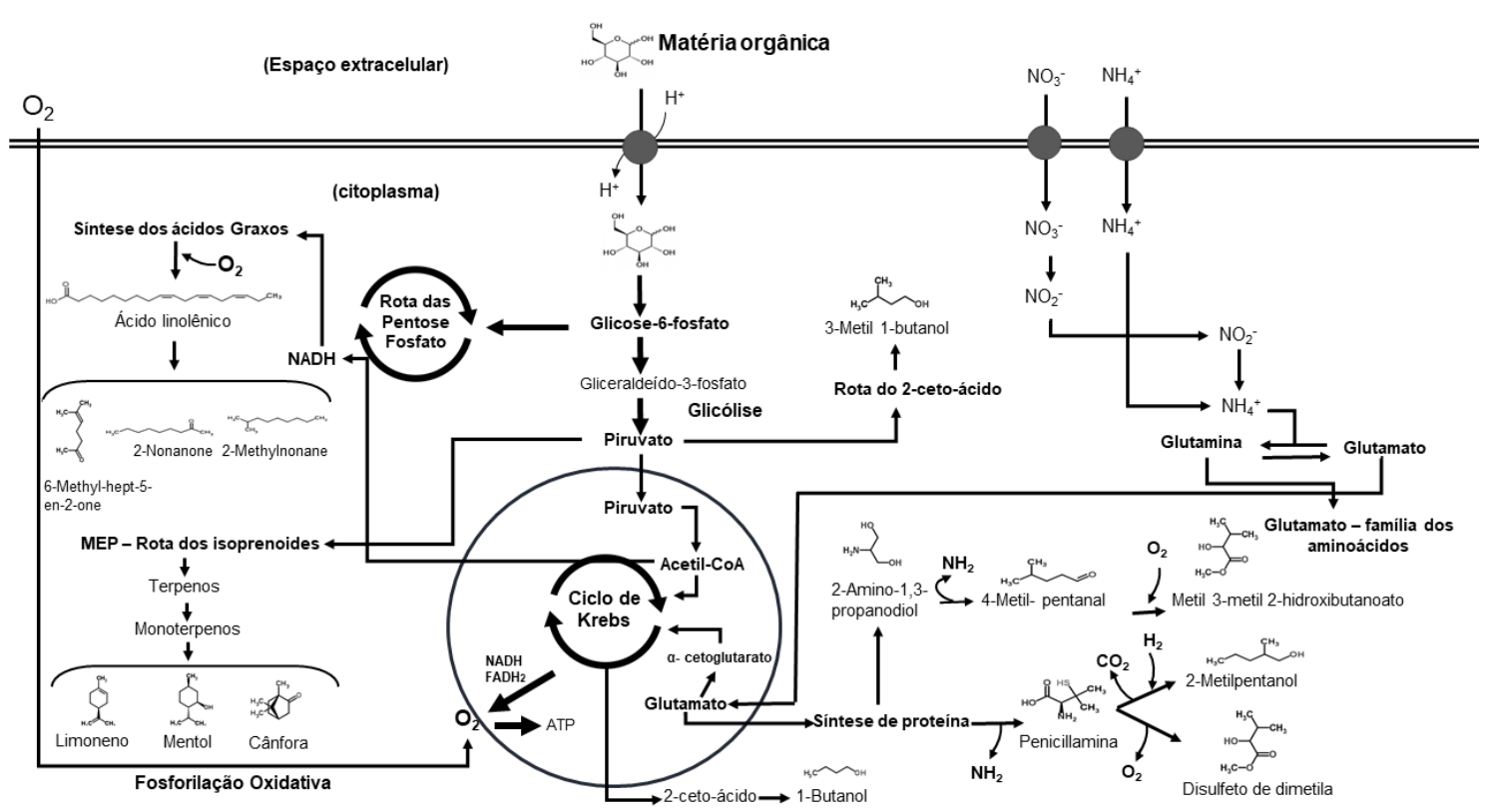

Figura 1. Rota metabólica de formação de compostos orgânicos voláteis por microalgas.

A via dos 2-cetoácidos é uma rota importante para a obtenção de compostos voláteis de diferentes classes químicas, como aldeídos, álcoois, ésteres e ácidos carboxílicos. Esta via envolve reações bioquímicas sequenciais, como extensão, descarboxilação, isomerização, redução, desidratação e esterificação de alguns aminoácidos de cadeia ramificada tais como, leucina e valina. Os compostos 1-butanol, 3-metil-butanal e 2-metil-butanal são reduzidos a 3-metil-butanol e 2-metil-butanol, além disso a reação pode ser estendida para 
formar 1-hexanol e outros álcoois (LIAO, PONTRELLI \& LUO, 2016; VIEIRA, PINHEIRO \& ZEPKA, 2020).

A via dos ácidos graxos começa com acetil-CoA usando malonil-CoA como bloco de construção, com base em uma série de reações cíclicas catalisadas pelo sistema multienzimático, denominado ácido graxo sintase. Compostos orgânicos voláteis como cetonas, aldeídos, hidrocarbonetos e álcoois podem ser produzidos a partir da degradação de ácidos graxos (SANTOS et al., 2016a; ZHOU, KERKHOVEN \& NIELSEN, 2018). Cetonas estruturalmente diversificadas são produtos metabólicos de ácidos graxos precursores (PINHEIRO et al., 2019; VIEIRA, PINHEIRO \& ZEPKA, 2020). Exemplos recentes incluem a produção de 2-heptanona e 2-nonanona a partir da oxidação do ácido linoléico (HAN et al., 2020).

Os aldeídos produzidos pela via dos ácidos graxos são aldeídos C6 e C9 que podem ser rapidamente metabolizados em álcoois por meio da enzima desidrogenase. Por exemplo, os ácidos graxos linoléico e ácido linolênico são conhecidos por serem precursores biossintéticos para 2,4-decadienal, 2heptanol, 2-octenal e 1-hexanal, que podem ser subsequentemente reduzidos a álcoois como 1-hexanol (BRAVO-LAMAS et al., 2018; JERKOVIĆ et al., 2018; PINHEIRO et al., 2019; VIEIRA, PINHEIRO \& ZEPKA, 2020).

A conversão do ácido graxo em hidrocarboneto ocorre usando aldeídos como substratos. Pelo menos duas enzimas, redutase de proteína transportadora acil-acil e oxigenase deformiladora de aldeído, são responsáveis por catalisar a reação (PINHEIRO et al., 2019; VIEIRA, PINHEIRO \& ZEPKA, 2020; BASRI et al., 2020).

Até o momento, três vias diferentes foram relatadas para sintetizar os isoprenóides: o ácido mevalônico (MVA); fosfato de metileritritol (MEP); e MVA modificado. No entanto, para espécies de microalgas, apenas as vias MVA / MEP foram descritas, ou ambas as vias em combinação (PINHEIRO et al., 2019; VIEIRA, PINHEIRO \& ZEPKA, 2020).

O isopentenil difosfato e o dimetilalil difosfato são os intermediários centrais do mecanismo dos isoprenóides. Em sequência, essas estruturas iniciais são transformadas em geranil difosfato seguido de farnesil difosfato (MEENA et al. 2017; PINHEIRO et al., 2019). Posteriormente, esses precursores de carbono são convertidos em terpenóides diversificados, por meio de uma 
série de reações catalisadas por três enzimas distintas: geranil difosfato sintase, farnesil difosfato sintase e geranilgeranil difosfato sintase, respectivamente. Finalmente, os carotenóides e seus produtos de clivagem oxidativa e enzimática são formados, como $\beta$-ionona e 6-metil-5-hepten-2-ona (DUDAREVA et al., 2013; SANTOS et al., 2016b).

Além disso, através do geranil difosfato como substrato de partida, são produzidos a geosmina e o 2-metilisoborneol (2-MIB) (Figura 2), no qual foram amplamente estudados devido a indesejáveis manifestações de sabor e odor. A síntese de 2-metilisoborneol, inicia com a metilação do precursor geranil difosfato em 2-metilgeranil difosfato que é ciclizado em 2-MIB (LEE et al., 2017). Em microalgas, a ciclização de farnesil difosfato pode formar geosmina, catalisada pela geosmina sintase por meio de três etapas (farnesil difosfato para germacradienol, germacradienol para 8,10-dimetil-1-octalina e 8,10-dimetil-1octalina para geosmina) (DURME et al., 2013; MEENA et al., 2017; LIATO \& AÏDER, 2017).

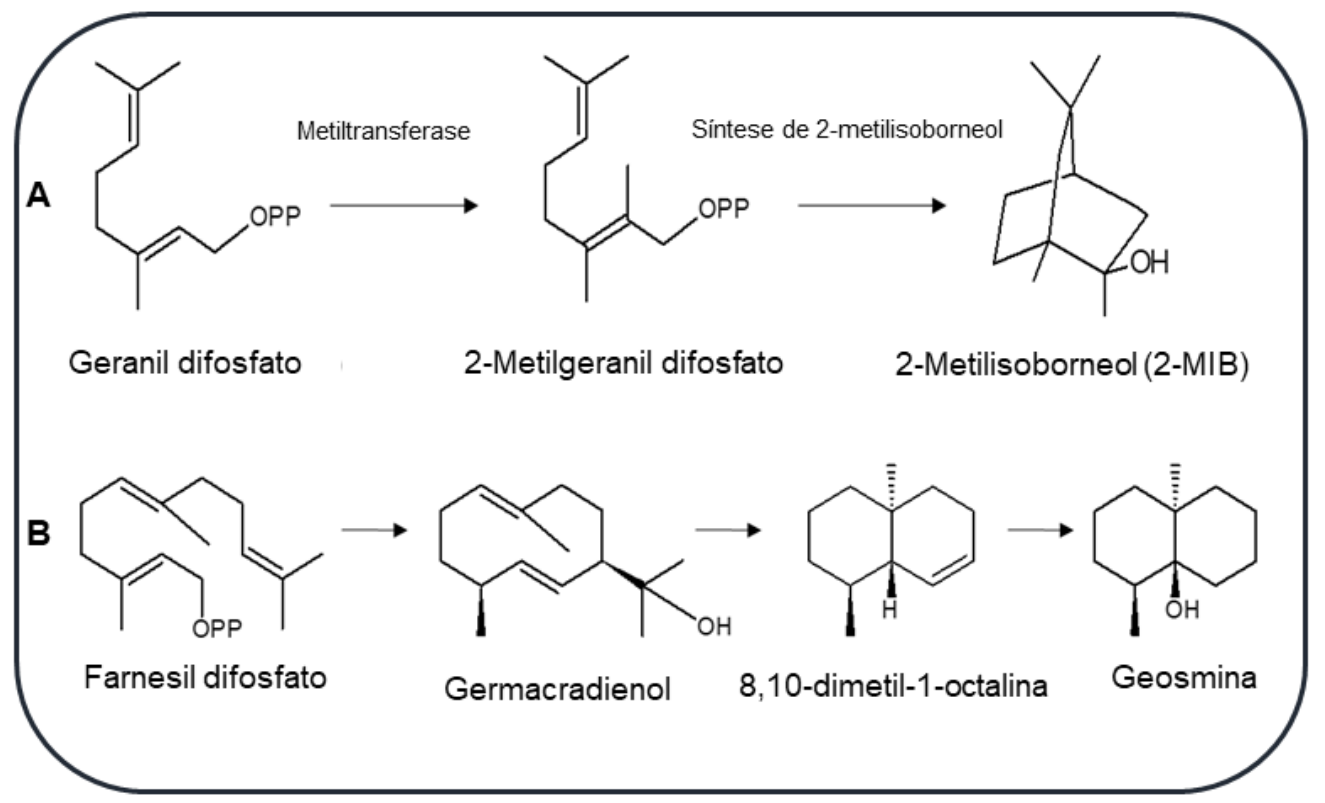

Figura 2. Mecanismo de formação de 2-MIB (A) e Geosmina (B).

As microalgas também liberam compostos de enxofre, como dimetilsulfeto, dimetildissulfeto e dimetiltrissulfeto, sendo que o sulfeto volátil mais importante produzido é dimetilsulfeto (WATSON \& JÜTTNER, 2017; ACHYUTHAN et al., 2017). Esses compostos podem ser derivados de 
aminoácidos, como a metionina, formando dimetilsulfoniopropionato (GIORDANO, M., \& PRIORETTI, 2014). A partir da desmetiolação do dimetilsulfoniopropionato, ele forma metanotiol que pode ser convertido em dimetilsulfeto por metilação (CURSON et al, 2017; ACHYUTHAN et al., 2017).

Os compostos de odor desejáveis e indesejáveis estão presente no meio ambiente, decorrentes de processos naturais e artificiais, pela geração de COVs (JACOB-LOPES \& FRANCO, 2013). Considerando a biossíntese de COVs pelas microalgas, embora dependente da espécie, sua produção pode ser modificada por vários fatores bióticos e abióticos, como fase de crescimento, estresses (temperatura, intensidade de luz, $\mathrm{pH}$, salinidade), nutrientes, gases $\left(\mathrm{H}_{2} \mathrm{O}, \mathrm{CO}_{2}\right.$, O3), aeração (mistura / turbulência) ou cultura estática (DURME et al., 2013; FRANCISCO et al., 2014; ACHYUTHAN et al., 2017). Assim, condições de cultivo controladas, podem influenciar na biotranformação, metabolização ou remoção de compostos voláteis do meio ambiente (VIEIRA et al., 2021).

\section{Desodorização de água residuária a partir de microalgas}

Microalgas, são consideradas como potenciais biocatalisadores para aplicação em processos biológicos de tratamento de água residuária industrial. Além disso, os sistemas de tratamento de efluentes empregando microalgas apresentam-se ambientalmente favoráveis com um carácter sustentável, uma vez que não geram poluentes adicionas, fornecendo oportunidades de reciclagem de nutrientes e conversão de resíduos orgânicos em uma infinidade de produtos, como, biocombustíveis, nutracêuticos, polímeros, pigmentos e variedades de produtos químicos biologicamente ativos para aplicações em diversos setores da biotecnologia (FRANCISCO et al., 2014; SANTOS et al., 2016a; WANG et al., 2017; LAURITANO et al., 2018).

Além disso, pesquisas atuais demonstraram a capacidade de microalgas em desodorizar água residuária de indústria frigorífica (VIEIRA et al., 2019; 2021). Águas residuais liberam uma variedade de grupos de poluentes atmosféricos que podem ser gerados em cada etapa do tratamento da água residual, causando a formação de precursores de compostos odoríferos com diferentes limear de percepção (LEBRERO et al., 2013; LEWKOWSKA et al., 2016). A Tabela 1 apresenta compostos orgânicos voláteis detectados em água residuária da indústria de processamento de carnes. 
Tabela 1. Compostos de odor de água residuária.

\begin{tabular}{|c|c|c|c|}
\hline Compostos & Fórmula química & $\operatorname{LP}^{a}\left(\mu \mathrm{g} \cdot \mathrm{m}^{-3}\right)$ & Descritor do odor ${ }^{a}$ \\
\hline dissulfeto de carbono & $\mathrm{CS}_{2}$ & $3 \times 10^{2}$ & desagradável, pútrido \\
\hline dimetil sulfeto & $\mathrm{C}_{2} \mathrm{H}_{6} \mathrm{~S}$ & $2 \times 10^{4}$ & repolho, sulfuroso \\
\hline 2-propenal & $\mathrm{C}_{3} \mathrm{H}_{4} \mathrm{O}$ & $7 \times 10^{2}$ & queimado \\
\hline 2-metilfurano & $\mathrm{C}_{5} \mathrm{H}_{6} \mathrm{O}$ & $3.5 \times 10^{3}$ & carne assada \\
\hline Butanal & $\mathrm{C}_{4} \mathrm{H}_{8} \mathrm{O}$ & $1.5 \times 10^{4}$ & doce \\
\hline 2-metilbutanais & $\mathrm{C}_{5} \mathrm{H}_{10} \mathrm{O}$ & $1 \times 10^{3}$ & amêndoa \\
\hline 3-metilbutanais & $\mathrm{C}_{5} \mathrm{H}_{10} \mathrm{O}$ & $2 \times 10^{2}$ & malte, óleo \\
\hline Tolueno & $\mathrm{C}_{7} \mathrm{H}_{8}$ & $5.95 \times 10^{5}$ & naftalina \\
\hline dissulfeto dimetil & $\mathrm{C}_{2} \mathrm{H}_{6} \mathrm{~S}_{2}$ & $3.5 \times 10^{3}$ & repolho podre, putrefação \\
\hline Hexanal & $\mathrm{C}_{6} \mathrm{H}_{12} \mathrm{O}$ & $2 \times 10^{2}$ & grama, gordura \\
\hline 1,4-cineole & $\mathrm{C}_{10} \mathrm{H}_{18} \mathrm{O}$ & $\mathrm{Na}^{b}$ & especiaria \\
\hline Limoneno & $\mathrm{C}_{10} \mathrm{H}_{16}$ & $1.7 \times 10^{3}$ & limão \\
\hline 1,8-cineole & $\mathrm{C}_{10} \mathrm{H}_{18} \mathrm{O}$ & $1.3 \times 10^{3}$ & especiaria \\
\hline 1 pentanol & $\mathrm{C}_{5} \mathrm{H}_{12} \mathrm{O}$ & $5 \times 10^{2}$ & frutado \\
\hline a-terpineno & $\mathrm{C}_{10} \mathrm{H}_{16}$ & na & limão \\
\hline$\rho$-cimeno & $\mathrm{C}_{10} \mathrm{H}_{14}$ & $7.1 \times 10^{3}$ & $\begin{array}{l}\text { limão, frutado, } \\
\text { combustível }\end{array}$ \\
\hline ciclohexanona & $\mathrm{C}_{6} \mathrm{H}_{10} \mathrm{O}$ & $3 \times 10^{2}$ & pimenta, acetona \\
\hline 2-heptanol & $\mathrm{C}_{7} \mathrm{H}_{16} \mathrm{O}$ & $1 \times 10^{5}$ & erva \\
\hline pirrolidina-2,4-diona & $\mathrm{C}_{4} \mathrm{H}_{5} \mathrm{NO}_{2}$ & na & na \\
\hline Hexanol & $\mathrm{C}_{6} \mathrm{H}_{14} \mathrm{O}$ & $1 \times 10^{1}$ & flor, verde \\
\hline dimetil trisulfide & $\mathrm{C}_{2} \mathrm{H}_{6} \mathrm{~S}_{3}$ & $1 \times 10^{2}$ & podre \\
\hline 1-heptanol & $\mathrm{C}_{7} \mathrm{H}_{16} \mathrm{O}$ & $2.5 \times 10^{6}$ & verde \\
\hline 3-propilciclopenteno & $\mathrm{C}_{8} \mathrm{H}_{14}$ & na & na \\
\hline benzaldeído & $\mathrm{C}_{7} \mathrm{H}_{6} \mathrm{O}$ & $1 \times 10^{1}$ & queimado \\
\hline Linalol & $\mathrm{C}_{10} \mathrm{H}_{18} \mathrm{O}$ & $1.4 \times 10^{2}$ & flor \\
\hline Fenchol & $\mathrm{C}_{10} \mathrm{H}_{18} \mathrm{O}$ & $5 \times 10^{4}$ & cânfora \\
\hline 4-terpineol & $\mathrm{C}_{10} \mathrm{H}_{18} \mathrm{O}$ & $3.4 \times 10^{-1}$ & mofo \\
\hline 2-octen-1-ol & $\mathrm{C}_{8} \mathrm{H}_{16} \mathrm{O}$ & $5 \times 10^{4}$ & sabão, plástico \\
\hline 1-nonanol & $\mathrm{C}_{9} \mathrm{H}_{20} \mathrm{O}$ & $5 \times 10^{1}$ & gordura \\
\hline fenilacetaldeído & $\mathrm{C}_{8} \mathrm{H}_{8} \mathrm{O}$ & $4 \times 10^{3}$ & mel \\
\hline acetofenona & $\mathrm{C}_{8} \mathrm{H}_{8} \mathrm{O}$ & $6.5 \times 10^{-1}$ & mofo, flor \\
\hline limonen-4-ol & $\mathrm{C}_{10} \mathrm{H}_{16} \mathrm{O}$ & na & menta fresca \\
\hline a-terpineol & $\mathrm{C}_{10} \mathrm{H}_{18} \mathrm{O}$ & $2.5 \times 10^{5}$ & óleo, hortelã \\
\hline álcool benzílico & $\mathrm{C}_{7} \mathrm{H}_{8} \mathrm{O}$ & $2 \times 10^{8}$ & doce, flor \\
\hline 2-feniletanol & $\mathrm{C}_{8} \mathrm{H}_{10} \mathrm{O}$ & $8.6 \times 10^{4}$ & flor \\
\hline o-cresol & $\mathrm{C}_{7} \mathrm{H}_{8} \mathrm{O}$ & $2 \times 10^{1}$ & medicinal, fenólico \\
\hline Fenol & $\mathrm{C}_{6} \mathrm{H}_{6} \mathrm{O}$ & $2 \times 10^{4}$ & borracha, medicinal \\
\hline$\rho$-cresol & $\mathrm{C}_{7} \mathrm{H}_{8} \mathrm{O}$ & $2 \times 10^{1}$ & sintético, fenol \\
\hline Indol & $\mathrm{C}_{8} \mathrm{H}_{7} \mathrm{~N}$ & $3 \times 10^{-1}$ & fecal \\
\hline Escatol & $\mathrm{C}_{9} \mathrm{H}_{9} \mathrm{~N}$ & $5.6 \times 10^{-4}$ & fecal \\
\hline
\end{tabular}

aLimiar de percepção

${ }^{b}$ na: não avaliado

Adaptado de Vieira et al., 2019. 
Estes COVs podem liberar uma variedade de odores que vai do agradável ao mais intenso e fétido. Assim, muitas são as tecnologias de redução de odor e são comumente classificadas em técnicas físicas, químicas e biológicas (ALFONSIN et al., 2015). A Norma Europeia 12255-9 (2002), recomenda a oxidação biológica, a oxidação química, a adsorção e oxidação térmica. No entanto, os métodos físicos e químicos de purificação de compostos de odor, apesar de comprovarem sua eficiência e confiabilidade e continuarem a ocupar seu nicho, ainda existem diversas desvantagens. Entre eles o alto custo de investimento e operação, além da possível geração de fluxos secundários de resíduos (BAJPAI, 2014; ALFONSIN et al., 2015).

Em contrapartida, os sistemas de tratamento biológico de odores utilizam processos bioquímicos para decompor compostos odoríferos. Estes métodos possuem a vantagem de converter os poluentes em produtos de oxidação como por exemplo, dióxido de carbono, água etc. São métodos de baixo custo, com simplicidade operacional e são considerados "tecnologias limpas", pois reduzem ou eliminam a necessidade de tratamento adicional dos produtos finais (BURGESS, PARSONS \& STUETZ, 2001; BAJPAI, 2014; CHENG et al., 2021).

Os métodos biológicos têm um amplo espectro de aplicações. São considerados os sistemas mais competitivos para a desodorização de poluentes do ar caracterizados por altas taxas de fluxo e baixas concentrações de contaminantes (SADDOUD \& SAYADI, 2007; VIKRANT et al., 2017). Além disso, o tratamento biológico é ambientalmente seguro, pois não produz compostos tóxicos prejudiciais à saúde ou ao meio ambiente. Geralmente é operado em condições naturais (temperatura e pressão atmosféricas normais).

Estes métodos para o tratamento de COVs incluem biofiltros, bioscrubbers (bio-lavador), biorreatores de membrana e filtros de biotrickling (filtro biológico percolador). Nestes métodos, os poluentes são degradados biologicamente por microrganismos aeróbios (VIKRANT et al., 2017). No entanto, estas tecnologias apesar de serem consideradas amigas da natureza, apresentam desvantagens como quedas excessivas de pressão, acúmulo gradual de subprodutos ácidos, dificuldade em controlar os parâmetros biológicos de operação, entupimento devido ao acúmulo de grande quantidade de biofilme e redução da eficiência do tratamento em altas concentrações de 
poluentes, além do alto custo de investimento (LEWKOWSKA et al., 2016; VIKRANT et al., 2017).

Assim, novas tecnologias de redução de odor têm sido amplamente investigadas como alternativas economicamente eficientes e confiáveis para a mitigação dos odores. Pesquisas utilizando bioprocessos baseados em microalgas demonstra ser uma tecnologia inovadora para desodorização de água residuária em estações de tratamento por ser uma alternativa econômica e ecologicamente correta, cuja flexibilidade metabólica é uma vantagem, pois converte moléculas polares e apolares de efluentes (VIEIRA et al., 2019; JACOBLOPES et al., 2020).

Bioprocessos baseado em microalgas no tratamento de controle de COVs desagradáveis encontrados em água residuária de processamento de aves e suínos, apresentaram uma eficiência de remoção de 99,6\% (VIEIRA et al., 2019). No entanto, ao ser realizado análise olfatométrica os pesquisadores constataram que não foi percebido nenhum descritor de odor desagradável após o período de 72 horas de tempo de residência celular, no qual concluíram que $100 \%$ dos compostos voláteis desagradáveis foram removidos da água residuária (VIEIRA et al., 2021).

Tecnologias de tratamento de odores em estações de tratamento de água residuária demonstraram que a eficiência de remoção de odores variou entre 70 a 95\% (LEBRERO et al., 2013). Pesquisas também mostraram uma eficiência de remoção de 99,7\% da emissão de odores de águas residuárias de suínos com o uso de células microbianas, no entanto o tempo de residência total foi de 260 horas (LOGAN et al., 2008). Assim, é possível perceber a capacidade do biorreator heterotrófico microalgal em mitigar os odores mais desagradáveis do efluente do processamento de carnes em um curto período de tempo.

As tecnologias atuais de controle de odor, ainda apresentam problemas para remover o indol e o escatol, ambos COVs são considerados um dos principais marcadores de odor de instalações de tratamento de água residuária do abate e processamento animal. Além disso, estes compostos estão presentes simultaneamente dos componentes estruturais hidrofóbicos e hidrofílicos (MATIAS et al., 2015; LEWKOWSKA et al., 2016). As microalgas apresentaram a capacidade em mitigar odores como indol e escatol, além da remoção de compostos hidrofóbicos, como terpenos e poluentes atmosféricos perigosos, 
como dissulfeto de carbono, acroleína, tolueno, acetofenona, o-cresol, fenol e $\rho$ cresol (EPA, 2008; VIEIRA et al., 2021).

Sistemas baseados em microalgas como uma tecnologia de tratamento de odor superam as desvantagens encontradas nas tecnologias já existentes. A tecnologia que utiliza microalgas, apesar de apresentar um alto custo inicial de implantação, não apresenta problemas de processo significativos. Além do mais, biorreatores microalgais podem ser utilizados em conjuntos com outras etapas de processo, como conversão de matéria orgânica e nutrientes da água residuária em uma infinidade de bioprodutos, reduzindo assim o custo de processo (SANTOS et al., 2016a; WANG et al., 2017; LAURITANO et al., 2018; DEPRÁ et al., 2019).

Apesar de estudos relatarem que as microalgas podem produzir compostos desagradáveis, como a presença de 2-MIB e geosmina (MEENA et al., 2017; LEE et al., 2017; LIATO \& AÏDER, 2017), estes compostos não foram detectados em pesquisas realizadas com a microalga Phormidium autumnale (SANTOS et al., 2016a; VIEIRA et al., 2019; 2021). Sabe-se que estes compostos podem ser facilmente liberados por muitas microalgas devido a fatores biótico e abióticos, no entanto, o uso de biorreatores facilitam o controle de temperatura e $\mathrm{pH}$, além do tempo de residência celular.

Vieira et al. (2021), demonstraram que sistemas baseados em microalgas além de biodegradar compostos de odor desagradável da água residuária, também conseguiu biotransformar e produzir novos compostos voláteis, maximizando o aproveitamento da biomassa microalgal, ao mesmo tempo que se reduz o impacto ambiental gerado por estes resíduos. Pesquisas com relação a COVs gerados por microalgas tem sido realizado atualmente, e estas apresentam um amplo espectro de classes de voláteis, podendo ser utilizadas como aromas, aditivos alimentares ou na geração de energia (SANTOS et al., 2016b; VIEIRA, PINHEIRO \& ZEPKA, 2020).

\section{Aplicação industrial de compostos orgânicos voláteis de microalgas}

As microalgas produzem uma variedade de COVs que podem ser aplicados como uma importante fonte alternativa de produtos químicos a granel e finos. Os compostos propanol, butanol, 3-metil-butanol, hexanol, hexanal, $\beta$ ciclocitral e $\beta$-ionona produzidos por microalgas possuem um apelo comercial 
(SANTOS et al., 2016b). Estes compostos de aromas gerados por microalgas podem competir com fontes tradicionais. A elucidação das vias metabólicas e precursores e a aplicação da bioengenharia convencional resultou em um conjunto de mais de 100 aromas químicos derivados da biotecnologia (BERGER, 2009).

O mercado global de compostos aromáticos deve atingir US $\$ 8,2$ bilhões em 2027, sendo os terpenos a classe de compostos predominante neste mercado (GLOBAL AROMA CHEMICALS MARKET, 2021). Outras classes também de grande interesse são os álcoois e os aldeídos, sendo importantes componentes do aroma amplamente aplicados em cosméticos, perfumaria e indústrias alimentícias (SANTOS et al., 2016b; PINHEIRO et al., 2019).

A identificação detalhada de tais compostos é muito importante devido aos seus impactos diretos nas propriedades aromáticas do produto final enriquecido com biomassa de microalgas (SANTOS et al., 2016a; ROBERTSON et al., 2016; ZHOU et al., 2017). Segundo Hosoglu (2018), compostos identificados como sendo responsáveis por tais características aromáticas são diferenciados em categorias tais como hidrocarbonetos, aldeídos, álcoois, ésteres, cetonas, e enxofre. Tabela 2 apresenta compostos voláteis detectado em microalgas.

Tabela 2. Compostos voláteis detectados em diferentes espécies de microalgas.

\begin{tabular}{|c|c|}
\hline $\begin{array}{l}\text { Compostos } \\
\text { voláteis }\end{array}$ & Microalgas \\
\hline \multicolumn{2}{|l|}{ Terpenos } \\
\hline a-ionona & $\begin{array}{l}\text { Botryococcus braunii, Rhodomonas sp., Tetraselmis chuii, } \\
\text { Nannochlopsis }\end{array}$ \\
\hline$\beta$-ciclocitral & $\begin{array}{l}\text { Botryococcus braunii, Chlorella vulgaris, Nannochloropsis oculata, } \\
\text { Nostoc sp., Phormidium autumnale, Rhodomonas sp., Spirulina } \\
\text { platensis, Tetraselmis chuii }\end{array}$ \\
\hline$\beta$-ionona & $\begin{array}{l}\text { Botryococcus braunii, Rhodomonas sp., Tetraselmis chuii, } \\
\text { Nannochlopsis, Spirulina platensis, Nostoc sp. }\end{array}$ \\
\hline geosmina & $\begin{array}{l}\text { Anabaena lemmermannii, Anabaena circinalis, Anabaena } \\
\text { solitaria, Anabaena viguieri, , Aphanizomenon gracile, } \\
\text { Geitlerinema splendidum, Leibleinia subtilis, Microcoleus sp., } \\
\text { Phormidium allorgei, Phormidium amoenum, Phormidium breve, } \\
\text { Phormidium cortianum, Phormidium formosum, Phormidium } \\
\text { simplicissimum Phormidium sp. }\end{array}$ \\
\hline
\end{tabular}


Oscillatoria curviceps, Oscillatoria limosa, Oscillatoria tenuis,

2-metilisoborneol

Oscillatoria variabilis, Phormidium autumnale, Phormidium breve, Phormidium calcícola, Phormidium favosum, Phormidium tenue, Phormidium sp.

geraniol

Synechococcus

mentol

Phormidium autumnale

citronelol

Oocystis pusilla

linalol

Chlorella sp., Chlamydomonas sp., Oocystis pusilla

\section{Aldeídos}

Benzaldeído

Botryococcus braunii, Rhodomonas sp., Tetraselmis chuii, Nannochloropsis oculata, Chlorella vulgaris, Nitzschia closterium.

Heptanal

Hexanal

2-metilpropanal

Botryococcus braunii, Rhodomonas sp., Thalassiosira weissflogii, Dicrateria inornata

Botryococcus braunii, Rhodomonas sp., Tetraselmis chuii, Nannochloropsis oculata, Chlorella vulgaris, Phormidium autumnale, Schizochytrium limacinum

Phormidium autumnale, Nannochloropsis oculata, Chaetoceros calcitrans, Thassiosira weissflogii, Platymonas helgolandica, Nitzschia closterium

3-metilbutanal

Rhodomonas sp., Tetraselmis chuii, Nannochloropsis oculata, Chlorella vulgaris, Phormidium autumnale

Botryococcus braunii, Rhodomonas sp., Nannochloropsis oculata, Chlorella vulgaris, Thalassiosira weissflogii, Nitzschia closterium,

nonanal Chaetoceros calcitrans, Platymonas helgolandica, Crypthecodinium cohnii, Schizochytrium limacinum, Chlorella prothecoides

Nitzschia closterium, Chaetoceros calcitrans, Thalassiosira 2,6-nonadienal weissflogii, Platymonas helgolandica, Nannochloropsis sp., Dicrateria inornata, Chlorella vulgaris

Botryococcus braunii, Nannochloropsis oculata, Thalassiosira

2-octenal weissflogii, Nitzschia closterium, Chaetoceros calcitrans, Dicrateria inornata

2-pentenal acetaldeído

Botryococcus braunii, Rhodomonas sp., Tetraselmis chuii, Nannochloropsis oculata, Chlorella vulgaris, Nitzschia closterium

Phormidium autumnale

\section{Sulfurados}

benzotiazol

Phormidium autumnale, Nitzschia closterium, Chaetoceros calcitrans, Thalassiosira weissflogii, Platymonas helgolandica, Nannochloropsis sp., Dicrateria inornata

dissulfeto dimetil

Rhodomonas sp., Tetraselmis chuii

dimetil sulfeto

Chaetoceros calcitrans, Chlorella protothecoides, Chlorella vulgaris, Crypthecodinium cohnii, Nannochloropsis sp., 
Oscillatoria chalybea, Oscillatoria tenuis, Phormidium autumnale, Plectonema boryanum, Synechococcus cedrorum, Tetraselmis chuii, Thalassiosira weissflogii

dimetil trisulfide

\section{Álcool}

Benzil álcool

cis-2-penten-1-ol

Etanol

1-hexanol

3-hexen-1-ol

2-etil-1-hexanol

ciclohexanol

isobutanol

2-metilbutanol

3-metilbutanol

1-octen-3-ol

2-feniletil álcool

1-pentanol

1-penten-3-ol

2-metil-1-

pentanol

\section{Hidrocarbonetos}

2,4-

dimetilheptano

dodecano

heptadecano
Rhodomonas sp., Tetraselmis chuii

Phormidium autumnale, Crypthecodinium cohnii, Schizochytrium limacinum, Chlorella prothecoides. Tetraselmis chuii, Nannochloropsis oculata, Chlorella vulgaris, Nitzschia closterium

Botryococcus braunii, Rhodomonas sp., Tetraselmis chuii, Nannochloropsis oculata, Chlorella vulgaris, Nitzschia closterium

Botryococcus braunii, Rhodomonas sp., Tetraselmis chuii, Nannochloropsis oculata, Chlorella vulgaris, Nitzschia closterium

Tetraselmis chuii, Nannochloropsis oculata, Chlorella vulgaris, Phormidium autumnale

Chlorella vulgaris

Tetraselmis chuii, Nannochloropsis oculata, Chlorella vulgaris, Nitzschia closterium, Spirulina platensis, Nostoc sp.

Phormidium autumnale

Phormidium autumnale

Tetraselmis sp.; Nannochloropsis, Chlorella vulgaris

Tetraselmis chuii, Nannochloropsis oculata, Chlorella vulgaris, Phormidium autumnale

Rhodomonas sp., Nannochloropsis oculata, Chlorella vulgaris, Crypthecodinium cohnii, Chlorella prothecoides, Tetraselmis chuii, Schizochytrium limacinum

Crypthecodinium cohnii

Botryococcus braunii, Rhodomonas sp., Tetraselmis chuii, Nannochloropsis oculata, Chlorella vulgaris, Nitzschia closterium

Botryococcus braunii, Rhodomonas sp., Tetraselmis chuii, Nannochloropsis oculata, Chlorella vulgaris, Nitzschia Closterium, Phormidium autumnale

Phormidium autumnale

Scenedesmus obliquus

Microcystis flos-aquae, Microcystis aeruginosa

Spirulina platensis, Nostoc sp., Nitzschia closterium, Chaetoceros calcitrans, Thalassiosira weissflogii, Platymonas helgolandica, 
Nannochloropsis sp., Dicrateria inornata, Microcystis flos-aquae, Microcystis aeruginosa

Spirulina platensis, Nostoc sp., Nitzschia closterium, Chaetoceros

hexadecano

pentadecano

tetradecano

tridecano

\section{Furanos}

2-etilfurano

2-pentilfurano

\section{Cetonas}

3-hidroxi-2-

butanona

acetil valeril

2,3-butanediona

2-heptanona

6-metil-5-hepten-

2-ona

2-octanediona

2-nonanona

3,5-octadien-2ona

2-propanona

2,3-pentenediona

1-penten-3-ona calcitrans, Thalassiosira weissflogii, Platymonas helgolandica,

Nannochloropsis sp., Dicrateria inornata, Microcystis flos-aquae,

Microcystis aeruginosa

Spirulina platensis, Nostoc sp., Nitzschia closterium, Chaetoceros calcitrans, Thalassiosira weissflogii, Platymonas helgolandica, Nannochloropsis sp., Dicrateria inornata

Spirulina platensis, Nostoc sp., Nitzschia closterium, Chaetoceros calcitrans, Thalassiosira weissflogii, Platymonas helgolandica, Nannochloropsis sp., Dicrateria inornata, Microcystis flos-aquae, Microcystis aeruginosa

Microcystis flos-aquae, Microcystis aeruginosa

Botryococcus braunii, Rhodomonas sp., Tetraselmis chuii, Nannochloropsis oculata, Chlorella vulgaris, Nitzschia closterium

Botryococcus braunii, Rhodomonas sp., Tetraselmis chuii, Nannochloropsis oculata, Chlorella vulgaris, Nitzschia closterium

Botryococcus braunii, Rhodomonas sp., Tetraselmis chuii, Nannochloropsis oculata, Chlorella vulgaris, Nitzschia closterium

Phormidium autumnale

Botryococcus braunii, Rhodomonas sp., Tetraselmis chuii, Nannochloropsis oculata, Chlorella vulgaris, Nitzschia closterium

Phormidium autumnale

Botryococcus braunii, Rhodomonas sp., Tetraselmis chuii, Nannochlopsis, Phormidium autumnale.

Botryococcus braunii, Rhodomonas sp., Tetraselmis chuii, Nannochloropsis oculata, Chlorella vulgaris, Nitzschia closterium

Phormidium autumnale

Botryococcus braunii, Rhodomonas sp., Tetraselmis chuii, Nannochloropsis oculata, Chlorella vulgaris, Nitzschia closterium, Chaetoceros calcitrans, Dicrateria inornata, Platymonas helgolandica

Scenedesmus obliquus

Botryococcus braunii, Rhodomonas sp., Tetraselmis chuii, Nannochloropsis oculata, Chlorella vulgaris, Nitzschia closterium

Botryococcus braunii, Rhodomonas sp., Tetraselmis chuii, Chlorella vulgaris, Nitzschia closterium, Chaetoceros calcitrans, Dicrateria inornata, Platymonas helgolandica 


\section{Ésteres}

metil octanoato

metil 3-metil 2-

hidroxibutanoato

metil penilacetato

2-metoxi-2metilpropano
Botryococcus braunii, Rhodomonas sp., Tetraselmis sp., Nannochlopsis, Crypthecodinium cohnii, Chlorella prothecoides, Tetraselmis chuii, Schizochytrium limacinum

\section{Phormidium autumnale}

Botryococcus braunii, Rhodomonas sp., Tetraselmis chuii, Nannochlopsis, Crypthecodinium cohnii, Chlorella prothecoides, Schizochytrium limacinum

Scenedesmus obliquus

Adaptado de Vieira, Pinheiro, Zepka et al., (2020).

A utilização da fração volátil da biomassa de microalgas pode representar uma melhoria na oferta para diferentes tipos de indústria. As microalgas são bem conhecidas pela capacidade de produção futura de biodiesel por apresentarem alta produtividade, biossíntese lipídica eficiente e praticamente não competem com as terras agricultáveis para a produção de alimentos (JACOB-LOPES et al., 2019). Também possuem robustez para capturar gases de efeito estufa, principalmente $\mathrm{O} \mathrm{CO}_{2}$ atmosférico, e se bioconverter em múltiplos bioprodutos. Esses excedentes provavelmente estarão disponíveis a um custo mínimo ou nenhum custo, o que favorece a abordagem da biorrefinaria de microalgas (DEPRÁ et al., 2018).

Os compostos orgânicos voláteis são considerados bioprodutos gasosos do metabolismo microalgal, podendo ser recuperados na forma de gases de exaustão. Esses sistemas podem produzir COVs com potencial energético. $O$ potencial de energia dos COVs produzidos no biorreator heterotrófico pode variar de $3,48 \times 10^{9}$ a $8,67 \times 10^{9} \mathrm{MJ} \mathrm{kg}^{-1}$, totalizando conteúdo de energia de 1,22 $\times$ $10^{13} \mathrm{MJ} \mathrm{kg}^{-1}$. Além disso, a taxa de geração de energia pode chegar a 1,01 $\times$ $10^{12} \mathrm{MJ} \cdot \mathrm{m}^{-3} \cdot \mathrm{d}^{-1}$ sob essas condições de cultivo (SEVERO et al., 2019; JACOBLOPES et al., 2020).

Considerando as estruturas químicas deste compostos, alguns álcoois apresentaram potencial energético comparável ao da gasolina (SANTOS et al., 2016b; PINHEIRO et al., 2019). Além disso, Halfmann, Gu e Zhou (2014), demonstraram que os compostos terpênicos possuem características atrativas 
como biodiesel e querosene de aviação. Da mesma forma, os hidrocarbonetos voláteis fornecem características de combustão desejáveis. Os aldeídos e cetonas podem ser considerados compostos intermediários de álcoois e hidrocarbonetos (MEENA et al., 2017; ACHYUTHAN et al., 2017).

\section{Conclusão}

Sistemas baseados em microalgas podem ser amplamente considerados como uma solução para resolver os diversos desafios da humanidade em relação aos problemas ambientais. As microalgas já são conhecidas por apresentarem potencial no processo de recuperação de efluentes, reduzindo o uso de energia de estratégias de gerenciamento de resíduos e regenerar nutrientes incluindo carbono, fósforo e nitrogênio. Alem disso, apresentam a capacidade de desodorizar água residuária, e simultaneamente produzir uma variedade de compostos voláteis de interesse comercial. A integração deste tipo de processo pode abordar as questões de sustentabilidade energética e reciclagem de resíduos no âmbito da bioeconomia circular, reduzindo os custos de produção de microalgas, aumentando a eficiência e a rentabilidade do processo. Embora o futuro para aplicações de sistemas baseados em microalgas pareça promissor, ainda há um longo caminho a ser percorrido para que se torne uma parte importante da indústria moderna.

\section{Agradecimentos}

Os autores agradecem ao Programa Nacional de Cooperação Acadêmica PROCAD/ CAPES, e ao Conselho Nacional de Desenvolvimento Científico e Tecnológico (CNPq).

\section{Referências}

ACHYUTHAN, K. E., HARPER, J. C., MANGINELL, R. P., MOORMAN, M. W. Volatile metabolites emission by in vivo microalgae-an overlooked opportunity? Metabolites, v.7, p.3, 2017. https://doi.org/10.3390/metabo7030039.

ALFONSÍN, C., LEBRERO, R., ESTRADA, J. M., MUNÕZ, R., KRAAKMAN, N.J.R., FEIJOO, G., MOREIRA, M.T. Selection of odour removal technologies in wastewater treatment plants: a guideline based on life cycle assessment. 
Journal of Environmental Management, v.149, p.77-84, 2015. https://doi.org/10.1016/j.jenvman.2014.10.011.

BAJPAI, P. Removal of Odours, in: Springer Briefs in Environmental Science, Biological Odour Treatment, 2014. https://doi.org/10.1007/978-3-31907539-6.

BASRI, R.S., ZALIHA, R.N., RAHMAN, R.A., KAMARUDIN, N.H.A., ALI, M.S.M. Cyanobacterial aldehyde deformylating oxygenase: Structure, function, and potential in biofuels production. International Journal of Biological Macromolecules, v.164, p.3155-3162, 2020.

BERENJIAN, A., CHAN, N., \& MALMIRI, H.J. Volatile organic compounds removal methods: a review. Journal Biochemistry, Biotechnology, v.8, p.220229, 2012. https://doi.org/10.3844/ajbbsp.2012.220.229.

BERGER, R.G. Biotechnology of flavours - the next generation. Biotechnology Letters, v.31, p.1651-1659, 2009. https://doi.org/10.1007/s10529-009-0083-5.

BRAVO-LAMAS, L., BARRON, L.J., FARMER, L., ALDAI, N. Fatty acid composition ofintramuscular fat and odour-active compounds of lamb commercialized in northern chromatography with mass spectrometry. Journal $\begin{array}{llll}\text { Chromatography } & \text { A } & \text { v.1350, } & \text { p.92-101, }\end{array}$ https://doi.org/10.1016/j.meatsci.2018.02.006.

BURGESS, J.E., PARSONS, S.A. \& STUETZ, R.M. Developments in odour control and waste gas treatment biotechnology: a review. Biotechnology Advances. v.19, p.35-63, 2001. https://doi.org/10.1016/S0734-9750(00)000586.

CALVIN, M., \& BENSON, A.A. The path of carbon in photosynthesis. Science, v.107, p.476-480, 1948. https://doi.org/10.1126/science.107.2784.476.

CAPELLI, L., SIRONI, S., DEL ROSSO, R., CÉNTOLA, P. Predicting odour emissions from wastewater treatment plants by means of odour emission factors.

Water Research, v.43, p.1977-1985, 2009.
https://doi.org/10.1016/j.watres.2009.01.022.

CHENG, H.H., LU, I.C., HUANG, P.W., WU, Y.J.; WHANG, L.M. Biological treatment of volatile organic compounds (VOCs)-containing wastewaters from 
wet scrubbers in semiconductor industry. Chemosphere, v.282, p.131137, 2021. https://doi.org/10.1016/j.chemosphere.2021.131137.

CURSON, A. R. J., LIU, J., BERMEJO MARTíNEZ, A., GREEN, R. T., CHAN, Y., CARRIÓN, O., WILLIAMS B. T., ZHANG, S. H., YANG, G. P., BULMAN PAGE, P. C., ZHANG, X. H., \& TODD, J. D. Dimethylsulfoniopropionate biosynthesis in marine bacteria and identification of the key gene in this process. Nature Microbiology, v.2, 2017. https://doi.org/10.1038/nmicrobiol.2017.9.

DEPRÁ, M.C., SANTOS, A.M., SEVERO, I.A., SANTOS, A.B., ZEPKA, L.Q., JACOB-LOPES, E. Microalgal biorefineries for bioenergy production: can we move from concept to industrial reality? BioEnergy Research, v.11, p.727-747, 2018. https://doi.org/10.1007/s12155-018-9934-z.

DEPRÁ, M.C.; MÉRIDA, L.G.R.; MENEZES, C.R.; ZEPKA, L.Q.; JACOBLOPES, E. A new hybrid photobioreactor design for microalgae culture. Chemical Engineering Research and Design, v.144, p.1-10, 2019. https://doi.org/10.1016/j.cherd.2019.01.023.

DOMENO, C., RODRíGUEZ-LAFUENTE, A., MARTOS, J.M., BILBAO, R., NERÍN, C. VOC removal and deodorization of effluent gases from an industrial plant by photooxidation, chemical oxidation, and ozonization. Environment $\begin{array}{lllll}\text { Science } \quad \& \quad \text { Technology, } & \text { v..44, } & \text { p.2585-2591, } & \end{array}$ https://doi.org/10.1021/es902735g.

DUDAREVA, N., KLEMPIEN, A., MUHLEMANN, J.K., KAPLAN, I. Biosynthesis, function and metabolic engineering of plant volatile organic compounds. New Phytologist, v.198, p.16-32, 2013. https://doi.org/10.1111/nph.12145.

DURME, J.V., GOIRIS, K., DE WINNE, A., DE COOMAN, L., MUYLAERT, K. Evaluation of the volatile composition and sensory properties of five species of microalgae. Journal of Agricultural and Food Chemistry, v.61, p.1088110890, 2013. https://doi.org/10.1021/jf403112k.

EN 12255-9:2002. European Standard on Wastewater treatment plants - Part 9: Odour control and ventilation. European Committee for Standardization (CEN), Brussels, January, 2002. 
FRANCISCO, É. C., FRANCO, T. T., WAGNER, R., \& JACOB-LOPES, E. Assessment of different carbohydrates as exogenous carbon source in cultivation of cyanobacteria. Bioprocess and Biosystems Engineering, v.37, p.14971505, 2014. https://doi.org/10.1007/s00449-013-1121-1.

GIORDANO, M., \& PRIORETTI, L. Sulphur and algae: metabolism, ecology and evolution. In: Borowitzka MA, Beardall J, Raven JA (eds) Microalgal physiology. Springer, Dordrecht, p.185-209, 2016. https://doi.org/10.1007/978-3319-24945-2_9.

GLOBAL AROMA CHEMICALS MARKET (2021 to 2026) - Industry Trends, Share, Size, Growth, Opportunity and Forecasts. July 02, 2021 08:33 ET | Source: Research and Markets, acessado em Agosto de 2021. https://www.globenewswire.com/news-

release/2021/07/02/2257148/28124/en/Global-Aroma-Chemicals-Market-2021to-2026-Industry-Trends-Share-Size-Growth-Opportunity-and-Forecasts.html.

HALFMANN, C., GU, L., \& ZHOU, R. Engineering cyanobacteria for the production of a cyclic hydrocarbon fuel from $\mathrm{CO}_{2}$ and $\mathrm{H}_{2} \mathrm{O}$. Green Chemistry, v.16, p.3175-3185, 2014. https://doi.org/10.1039/c3gc42591f.

HAN, G., ZHANG, L., LI, Q., WANG, Y., CHEN, Q., KONG, B. Impacts of different altitudes and natural drying times on lipolysis, lipid oxidation and flavour profile of traditional Tibetan yak jerky. Meat Science, v.162, p.108030, 2020. https://doi.org/10.1016/j.meatsci.2019.108030.

HERRERO, A., MURO-PASTOR, A. M., FLORES, E. Nitrogen control in cyanobacteria. Journal of bacteriology, v.183, p.411-425, 2001. https://doi.org/10.1128/JB.183.2.411-425.2001.

HOSOGLU, M. I. Aroma characterization of five microalgae species using solidphase microextraction and gas chromatography-mass spectrometry/olfactometry. Food Chemistry, v.240, p.1210-1218, 2018. https://doi.org/10.1016/j.foodchem.2017.08.052.

JACOB-LOPES, E., \& FRANCO, T. T. From oil refinery to microalgal biorefinery. Journal of $\mathrm{CO}_{2} \quad$ Utilization, $\quad$ v.2, $\quad$ p.1-7, 2013. https://doi.org/10.1016/j.jcou.2013.06.001. 
JACOB-LOPES, E., MARONEZE, M.M., DEPRÁ, M.C., SARTORI, R.B., DIAS, R.R., ZEPKA, L.Q. Bioactive food compounds from microalgae: An innovative framework on industrial biorefineries. Current Opinion in Food Science. In Press. v.25, p.1-7, 2019. https://doi.org/10.1016/j.cofs.2018.12.003.

JACOB-LOPES, E., SANTOS, A. B., SEVERO, I. A., DEPRÁ, M. C., MARONEZE, M. M., \& ZEPKA, L. Q. Dual production of bioenergy in heterotrophic cultures of cyanobacteria: Process performance, carbon balance, biofuel quality and sustainability metrics. Biomass and Bioenergy, 142, 2020. https://doi.org/10.1016/j.biombioe.2020.105756.

JERKOVIĆ, I., MARIJANOVIĆ, Z., ROJE, M., KÚS, P.M., JOKIĆ, S., ČOŽRAKOVAC, R. Phytochemical study of the headspace volatile organic compounds of fresh algae and seagrass from the Adriatic Sea (single point collection). Plos One. v.13, p.1-13, 2018. https://doi.org/10.1371/journal.pone.0196462.

KONG, W., SHEN, B., LYU, H., KONG, J., MA, J., WANG, Z., FENG, S. Review on carbon dioxide fixation coupled with nutrients removal from wastewater by microalgae. Journal of Cleaner Production, v.292, p.125975, 2021. https://doi.org/10.1016/j.jclepro.2021.125975.

LAURITANO, C., MARTIN, J., CRUZ, M., REYES, F., ROMANO, G., LANORA, A. First identification of marine diatoms with anti-tuberculosis activity. Science Reports, v.8, p.1-10, 2018. https://doi.org/10.1038/s41598-018-20611-x.

LEBRERO, R., VOLCKAERT, D., PÉREZ, R., MUNÕZ R., VAN LANGENHOVE, $\mathrm{H}$. A membrane bioreactor for the simultaneous treatment of acetone, toluene, limonene and hexane at trace level concentrations. Water Research, v.47, 21992212, 2013. https://doi.org/10.1016/j.watres.2013.01.041.

LEE, J., RAI, P.K., JEON, Y.J., KIM, K.H., KWON, E.E. The role of algae and cyanobacteria in the production and release of odorants in water. Environmental Pollution, v.227, p.252-262, 2017. https://doi.org/10.1016/j.envpol.2017.04.058. LEWKOWSKA, P., CIEŚLIK, B., DYMERSKI, T., KONIECZKA, P., \& NAMIEŚNIK, J. Characteristics of odors emitted from municipal wastewater treatment plant and methods for their identification and deodorization techniques. 
Environmental

Research,

151,

573-586,

2016.

https://doi.org/10.1016/j.envres.2016.08.030.

LIAO, J. C., MI, L., PONTRELLI, S., \& LUO, S. Fuelling the future: Microbial engineering for the production of sustainable biofuels. Nature Reviews Microbiology, 14(5), 288-304, 2016. https://doi.org/10.1038/nrmicro.2016.32.

LIATO, V., \& AIIDER, M. Geosmin as a source of the earthy-musty smell in fruits, vegetables and water: Origins, impact on foods and water, and review of the removing techniques. Chemosphere, v.181, p.9-18, 2017. https://doi.org/10.1016/j.chemosphere.2017.04.039.

LOGAN, B.E., D. CALL, D., S. CHENG, S., HAMELERS, H.V.M., SLEUTELS, A.W. JEREMIASSE, T.H.J.A., ROZENDAL, R.A. Microbial electrolysis cells for high yield hydrogen gas production from organic matter. Environmental Science Technology, v.42, p.8630-8640, 2008. https://doi.org/10.1021/es801553z.

LOURENÇO, S. O. Cultivo de Microalgas Marinhas: Princípios e Aplicações. Editora RiMa, p.51-60, 2006.

MATIAS, T., MARQUES, J., QUINA, M.J., GANDO-FERREIRA, L., VALENTE, A.J.M., PORTUGAL, A., DURÃES, L. Silica-based aerogels as adsorbents for phenol-derivative compounds. Colloids and Surfaces A: Physicochemical and Engineering Aspects, v.480, p.260-269, 2015. https://doi.org/10.1016/j.colsurfa.2015.01.074.

MEENA, S., KUMAR, S.R., DWIVEDI, V., KUMAR SINGH, A.K., CHANOTIYA, C.S., AKHTAR, Q., KUMAR, K., SHASANY, A.K., NAGEGOWDA, D.A. Transcriptomic insight into terpenoid and carbazole alkaloid biosynthesis, and functional characterization of two terpene synthases in curry tree (Murraya koenigii). Science Repports, v.7, p.44126, 2017. https://doi.org/10.1038/srep44126.

MOORE, J.P. (Ed.), Volatile organic compounds: occurrence, behavior and ecological implications, ed. Nova Science Publishers, New York, pp. 1-18, 2001.

MUÑOZ, R., MALHAUTIER, L., FANLO, J.L., QUIJANO, G. Biological technologies for the treatment of atmospheric pollutants. International Journal 
Environmental Analytical Chemistry, v.95, p.950-967, 2015. https://doi.org/10.1080/03067319.2015.1055471.

NASCIMENTO, T.C.; CAZARIN, C.B.B., MARÓSTICA JR, M.R.; RISSO, E.M.; AMAYA-FARFAN, J.; GRIMALDI, R.; MERCADANTE, A.Z.; JACOB-LOPES, E., ZEPKA, L.Q. Microalgae biomass intake positively modulates serum lipid profile and antioxidant status. Journal of Functional Foods, v.58, p. 11-20, 2019. https://doi.org/10.1016/j.jff.2019.04.047.

NOREÑA-CARO, D \& BENTON, M.G. Cyanobacteria as photoautotrophic biofactories of high-value chemicals. Journal of $\mathrm{CO}_{2}$ Utilization, v.28, p.335366, 2018. https://doi.org/10.1016/j.jcou.2018.10.008.

PEREZ-GARCIA, \& BASHAN. Algal biorefineries: Volume 2: Products and refinery design. In Algal Biorefineries: Volume 2: Products and Refinery Design, 2015. https://doi.org/10.1007/978-3-319-20200-6.

PINHEIRO, P. N., VIEIRA, K. R., SANTOS, A. B., JACOB-LOPES, E., ZEPKA, L. Q. Biogeneration of Volatile Organic Compounds in Microalgae-Based Systems. In: Gokare A. Ravishankar and Ranga Rao Ambati. (Org.). Handbook of Algal Technologies and Phytochemicals - Food, Health and Nutraceutical Applications. 1ed.Boca Raton: CRC Press, 2019, v. 1, p. 100-110.

POVEDA, J. Beneficial effects of microbial volatile organic compounds (MVOCs). $\begin{array}{lllll}\text { Applied } & \text { Soil } & \text { Ecology, } & \text { v.168, } & \text { p.104118, }\end{array}$ https://doi.org/10.1016/j.apsoil.2021.104118.

ROBERTSON, R.C.; MATEO, M.R.G.; O'GRADY, M.N.; GUIHENEUF, F.; STENGEL, D.B.; ROSS, R.P.; FITZGERALD, G.F.; KERRY, J.P.; STANTON, C. An assessment of the techno-functional and sensory properties of yoghurt fortified with a lipid extract from the microalga Pavlova lutheri Innovative. Food Science and Emerging Technologies, v.37, p.237-246, 2016.

SADDOUD, A., \& SAYADI, S. Application ofacidogenic fixed-bed reactor prior to anaerobic membrane bioreactor for sustainable slaughterhouse wastewater treatment. Journal of Hazardous Materials, v.149, p.700-706, 2007. https://doi.org/10.1016/j.jhazmat.2007.04.031. 
SANTOS, A. B., FERNANDES, A. S., WAGNER, R., JACOB-LOPES, E., ZEPKA, L. Q. Biogeneration of volatile organic compounds produced by Phormidium autumnale in heterotrophic bioreactor. Journal of Applied Phycology. v.28, p.1561-1570, 2016a. https://doi.org/10.1007/s10811-015-0740-0.

SANTOS, A.B., VIEIRA, K.R., NOGARA, G.P., WAGNER, R., JACOB-LOPES, E., ZEPKA, L.Q. Biogeneration of Volatile Organic Compounds by Microalgae/cyanobacteria: Occurrence, Be-havior, Ecological Implications and Industrial Applications. In: Moore, J. P. (Org.). Volatile Organic Compounds: Occurrence, Behavior and Ecological Implications. 1ed.: Nova Science Publishers. New York. Current Biotecnology, v.4, p.249-254, 2016b.

SANTOS, A.M., SANTOS, A. M., SARTORI, R.B., QUEIROZ, L. Z., BARIN, J. S., JACOB-LOPES, E., Nutrient cycling in meat processing industry by microalgae/cyanobacteria-based processes. Desalination and Water Treatment. v.100, p.91-99, 2017. https://doi.org/10.5004/dwt.2017.21719.

SEVERO, I.A., DEPRÁ, M.C., DIAS, R.R., JACOB-LOPES, E. Process integration applied to microalgae-based systems. Handbook of MicroalgaeBased Processes and Products, v.1, p.709-735, 2020. https://doi.org/10.1016/b978-0-12-818536-0.00026-9.

SEVERO, I.A., PINHEIRO, P.N., VIEIRA, K.R., ZEPKA, L.Q., JACOB-LOPES, E. Biological conversion of carbon dioxide into volatile organic compounds. In: Inamuddin et al. (Eds.), Conversion of carbon dioxide into hydrocarbons: v.2, ed. Springer, Cham, p. 45-73, 2020. https://doi.org/10.1007/978-3-030-286385_2.

SEVERO, I.A., SIQUEIRA, S.F., DEPRÁ, M.C., MARONEZE, M.M., ZEPKA, L.Q., JACOB-LOPES, E. Biodiesel facilities: What can we address to make biorefineries commercially competitive? Renewable \& Sustainable Energy Reviews, v.112, p.686-705, 2019. https://doi.org/10.1016/j.rser.2019.06.020.

SU, Y. Revisiting carbon, nitrogen, and phosphorus metabolisms in microalgae for wastewater treatment. Science of the Total Environment, v.762, p.144590, 2021. https://doi.org/10.1016/j.scitotenv.2020.144590.

SUGANYA, T., VARMAN, M., MASJUKI, H.H., RENGANATHAN, S. Macroalgae and microalgae as a potential source for commercial applications along with 
biofuels production: A biorefinery approach. Renewable \& Sustainable Energy Reviews. v.55, p.909-941, 2016. https://doi.org/10.1016/j.rser.2015.11.026.

US EPA, Initial List of Hazardous Air Pollutants with Modifications, U.S. Environmental Protection Agency, Washington, D.C., 2008.

VIEIRA, K. R., PINHEIRO, P. N. \& ZEPKA, L. Q. Volatile organic compounds from microalgae. In Handbook of Microalgae-Based Processes and Products. Elsevier Inc. 2020. https://doi.org/10.1016/b978-0-12-818536-0.00024-5.

VIEIRA, K. R., PINHEIRO, P. N., SANTOS, A. B., CICHOSKI, A. J., MENEZES, C. R., WAGNER, R., ZEPKA, L. Q., \& JACOB-LOPES, E. The role of microalgaebased systems in the dynamics of odors compounds in the meat processing industry. Desalination and Water Treatment. v.150, p.282-292, 2019. https://doi.org/10.5004/dwt.2019.23730.

VIEIRA, K.R., MARONEZE, M.M., KLEIN, B., WAGNER, R., QUEIROZ, M.I., JACOB-LOPES, E., ZEPKA, L.Q., The role of microalgae-based systems in the dynamics of odorous compounds in the meat processing industry. Part II Olfactometry and sensory relevance. Desalination and Water Treatment. v.232, p.16-25, 2021. https://doi.org/10.5004/dwt.2021.27458.

VIKRANT, K., KIM, K. H., SZULEJKO, J. E., PANDEY, S. K., SINGH, R. S., GIRI, B. S., BROWN, R. J. C., \& LEE, S. H. Bio-filters for the treatment of VOCs and odors - a review. Asian Journal of Atmospheric Environment, v.11, p.139-152, 2017. https://doi.org/10.5572/ajae.2017.11.3.139.

WANG, X., BAO, K., CAO, W., ZHAO, Y., HU, W.C. Screening of microalgae for integral biogas slurry nutrient removal and biogas upgrading by different microalgae cultivation technology. Scientific Reports, v.7, p.1-12, 2017. https://doi.org/10.1038/s41598-017-05841-9.

WANG, X., BAO, K., CAO, W., ZHAO, Y., HU, W.C. Screening of microalgae for integral biogas slurry nutrient removal and biogas upgrading by different microalgae cultivation technology. Science Repports, v.7, p.1-12, 2017. https://doi.org/10.1038/s41598-017-05841-9.

WATSON, S. B., \& JÜTTNER, F. Malodorous volatile organic sulfur compounds: Sources, sinks and significance in inland waters. Critical Reviews in 
Microbiology,

v.43,

p.210-237,

2017.

https://doi.org/10.1080/1040841X.2016.1198306.

ZARGAR, A., BAILEY, C.B., HAUSHALTER, R.W., EIBEN, C.B., KATZ, L., KEASLING, J.D. Leveraging microbial biosynthetic pathways for the generation of 'drop-in' biofuels. Current Opinion in Biotechnology, v.45, p.156-63, 2017. https://doi.org/10.1016/j.copbio.2017.03.004.

ZHOU, L., CHEN, J., XU, J., LI, Y., ZHOU, C., YAN, X. Change of volatile components in six microalgae with different growth phases. Journal of the Science of Food and Agriculture, v.97, p.761-769, 2017. https://doi.org/10.1002/jsfa.7794.

ZHOU, Y. J., KERKHOVEN, E. J., \& NIELSEN, J. Barriers and opportunities in bio-based production of hydrocarbons. Nature Energy, v.3, p.925-935, 2018. https://doi.org/10.1038/s41560-018-0197-x.

\section{Autores}

Karem Rodrigues Vieira*, Pricila Nass Pinheiro, Tatiele Casagrande do Nascimento

Departamento de Ciência e Tecnologia de Alimentos, Universidade Federal de Santa Maria, Avenida Roraima, 1000, 97105-900, Santa Maria, RS, Brasil.

\footnotetext{
*Autor para correspondência: merakvieira@gmail.com
} 